\title{
GENERALIZED INTRINSIC FORM OF THE CHARAGTERISTIC RELATIONS IN THE STEADY SUPERSONIC FLOW OF A GAS
}

\author{
E. R. SURYANARAYAN
}

(Received 10 November 1966, revised 11 July 1967)

\section{Introduction}

Coburn [1] has derived the intrinsic form of the characteristic relations, for the steady, supersonic, three-dimensional motion of a polytropic gas. The purpose of this paper is to obtain a generalized form of these relations and to apply them to obtain two classes of complex-screw motions [2].

The generalized characteristic relations are derived by the use of the substitution principle [3]. This principle has been used by Neményi and Prim [4], Hansen and Martin [5] to study the geometrical properties of plane flows. Smith [6], [7] has studied the intrinsic properties of spatial flows and some properties of magnetohydrodynamic flows by the use of this principle. In this paper the equations of motion, continuity and energy are transformed to the reduced system by the substitution principle, and it is shown that the reduced system and the original system have the same cone of normals.

The generalized characteristic relations are obtained by expressing the reduced system of equations governing the flow in terms of a set of characteristic variables.

The classes of fluid motions to be studied are characterized by the fact that the right circular helices form the normal congruence to $\infty^{1}$ characteristic surfaces. Two distinct classes of flows are obtained; one for which the bicharacteristic direction is along the binormal to the characteristic curve (normal to the characteristic surface), and the other for which bicharacteristic direction is along the radius of the cylinder. These classes of flows are rotational. The stream lines of the first class of flows are right circular helices; and the stream lines of the second class of flows lie on a right circular helicoid [8].

\section{Basic equations}

Let $x^{j}(j=1,2,3)$ denote a Cartesian orthogonal coordinate system in $E^{3}$, a three dimensional Euclidean space, and let us denote the partial derivative by the symbolism 


$$
\partial_{j}=\frac{\partial}{\partial x^{j}} .
$$

In $E^{3}$ covariant and contravariant quantities are equivalent. However, in order to use the summation convention, we shall write the indices in covariant and contravariant positions. Let $g_{i j}$ denote the fundamental tensor of $E^{3}$.

The equations governing the flow of a stationary polytropic gas, neglecting viscosity and thermal conductivity, are

$$
\begin{aligned}
\partial_{j}\left(\rho u^{j}\right) & =0, \\
\rho u^{j} \partial_{j} u_{i}+\partial_{i} p & =0, \\
u^{j} \partial_{j} \eta & =0, \\
\rho & =P(p) S(\eta),
\end{aligned}
$$

where $u_{j}$ are the components of the velocity, $\rho$ is the density, $p$ is the pressure, $\eta$ denotes the specific entropy and

$$
P(p)=p^{1 / \gamma}
$$

$\gamma$ being the adiabatic exponent. For a polytropic gas the equation of motion (2.2) can be written [9]

$$
\gamma \rho u^{j} \partial_{j} u_{k}+\partial_{k}\left(\rho c^{2}\right)=0 .
$$

Here, $c$ is the local sound speed defined by

$$
c^{2}=\left(\frac{\partial p}{\partial \rho}\right)_{\eta}=\frac{\gamma p}{\rho} .
$$

In addition we shall use as the energy relation, the Bernoulli equation

$$
u^{j} \partial_{j}\left(\frac{c^{2}}{\gamma-1}+\frac{u^{2}}{0}\right)=0,
$$

where $u$ is the magnitude of the velocity. The above condition is equivalent to the fact that the entropy remains constant along the stream line.

By definition, the characteristic manifolds of the system (2.1), (2.6) and $(2.8)$ are the surfaces along which the discontinuities of the derivatives $\partial_{j} \rho, \partial_{j} c, \partial_{j} u_{k}$ can occur. By eliminating $\rho$ between (2.1) and (2.6) and then eliminating $\partial_{j} c^{2}$ in the resulting equation by use of $(2.8)$, we obtain the following relation

$$
\left(u^{j} u^{k}-c^{2} g^{j k}\right) \partial_{j} u_{k}=0 .
$$

For supersonic flows it can be shown that the above equation is hyperbolic. If 


$$
a^{j k}=u^{j} u^{k}-c^{2} g^{j k}
$$

then the characteristic manifolds of $(2.9)$ are determined by the cone

$$
a^{j k} n_{j} n_{k}=0,
$$

where $n_{j}$ is the unit vector along the normal to one of the characteristic surfaces [10]. From the theory of the characteristic manifolds it is known that for supersonic flows, the velocity vector lies along the axis of the normal cone and is given by [1]

$$
u^{j}=c n^{j}+b t^{j},
$$

where $t^{j}$ is a unit vector along the bicharacteristic curves, $b$ and $c$ are positive, and

$$
b^{2}=u^{2}-c^{2}
$$

\section{The characteristic manifolds of the substitute flow}

Using the substitution principle [3], we can define new velocity components $u_{*}^{i}$, density $\rho_{*}$, entropy $\eta_{*}$ by

$$
\alpha u_{*}^{i}=u^{i}, \quad \rho_{*}=\alpha^{2} \rho, \quad S\left(\eta_{*}\right)=\alpha^{2} S(\eta),
$$

$\alpha$ being a scalar function remaining constant along a stream line, that is,

$$
u^{j} \partial_{j} \alpha=0, \quad \alpha \neq 0 .
$$

The pressure remains invariant under this transformation. The equations (2.1), (2.6) and (2.8) of the flow $F$ by virtue of (3.1), now become

$$
\begin{aligned}
\partial_{j}\left(\rho_{*} u_{*}^{j}\right) & =0, \\
\gamma \rho_{*} u_{*}^{j} \partial_{j} u_{* k}+\partial_{k}\left(\rho_{*} c_{*}^{2}\right) & =0, \\
u_{*}^{j} \partial_{j}\left(\frac{c_{*}^{2}}{\gamma-1}+\frac{u_{*}^{2}}{2^{2}}\right) & =0 .
\end{aligned}
$$

Here $u_{*}$ is the magnitude of the reduced velocity $u_{*}^{i}$ and $c_{*}$ is the reduced sound speed defined by

$$
c=\alpha c_{*} .
$$

The substitute flow $F_{*}$ given by the equation (3.3)-(3.5) has the same stream line as the original flow $F$. If $F_{*}$ is irrotational, $F$ in general is rotational. If $M$ and $M_{*}$ denote the Mach numbers of the two flows, we find from the first equation of (3.1) and (3.6) that

$$
M=\frac{u}{c}=\frac{u_{*}}{c_{*}}=M_{*} .
$$


Therefore the Mach number remains invariant under the substitution principle [3].

To obtain the generalized characteristic relations, we define the generalized characteristic manifolds of $F_{*}$-system (3.3)-(3.5), to be those surfaces along which the discontinuities of the derivatives of $\partial_{j} \rho_{*}, \partial_{j} c_{*}$, $\partial_{j} u_{* k}$ can occur. By eliminating $\rho^{*}$ between (3.3) and (3.4) and then by eliminating $\partial_{j} c_{*}^{2}$ in the resulting equation by use of (3.5) we obtain

$$
\left(u_{*}^{j} u_{*}^{k}-c_{*}^{2} g^{k j}\right) \partial_{j} u_{* k}=0 .
$$

If the symmetric tensor $a_{*}^{j k}$ is defined by

$$
a_{*}^{j k}=u_{*}^{j} u_{*}^{k}-c_{*}^{2} g^{j k}
$$

then the characteristic manifolds of (3.8) are determined by the cone

$$
a_{*}^{j k} n_{*_{j}} n_{* k}=0 \text {, }
$$

where $n_{*}^{j}$ is the unit vector along the normal to one of the generalized characteristic surfaces. Since (3.8) reduces to (2.9) by virtue of the first equation of (3.1), the equation (3.8) is also hyperbolic. The velocity $u_{*}^{i}$ lies along the axis of the normal cone and is given by

$$
u_{*}^{j}=c_{*} n_{*}^{j}+b_{*} t_{*}^{j} .
$$

Here $t_{*}^{j}$ is a unit vector along the bicharacteristic curves of the equation (3.8), $b_{*}$ is positive and

$$
b_{*}^{2}=u_{*}^{2}-c_{*}^{2} .
$$

Let us now compare the geometric properties of the characteristic systems $F$ and $F_{*}$. From (3.9), (3.6), the first equation of (3.1) and (2.10), we find that

$$
a^{j k}=\alpha^{2} a_{*}^{j k}
$$

and from (3.10) and (3.13),

$$
a^{j k} n_{* j} n_{* k}=0 .
$$

From (2.11) and (3.14) we see that $n_{*}^{j}$ lies on the characteristic cone of the system $F$. From (2.12), (3.11), and the first equation of (3.1), we find that

$$
u^{j}=c n_{*}^{j}+b t_{*}^{j} .
$$

Since $n_{*}^{j}$ lies on the characteristic cone of $F$, we may take

$$
n_{*}^{j}=n^{j}
$$

and (3.15), (3.16) and (2.12) now imply that 


$$
t^{j}=t_{*}^{j} .
$$

Therefore, the bicharacteristic curves of $F$ and $F_{*}$ coincide. Hence we have the theorem: The substitute flow has the same cone of normals, characteristic manifolds and bicharacteristic curves as the original flow.

\section{The generalized characteristic relations}

Since the characteristic manifolds and bicharacteristic curves of the two flows $F$ and $F_{*}$ coincide, let $n^{j}$ denote the unit vector along the normal to the characteristic manifolds and $t^{j}$ be the unit vector along the bicharacteristic curves. Let $s^{j}$ denote a unit vector which is orthogonal to both $t^{j}$ and $n^{j}$, so that $s^{j}, t^{j}$ and $n^{j}$ form an ordered orthogonal right handed triple at each point. The curvature vector of the $n^{j}$ congruence will be denoted by $v^{j}$, the curvature vector of $s^{j}$ congruence will be denoted by $\sigma^{j}$ and the curvature vector of $t^{j}$ congruence will be denoted by $\tau^{j}$ so that

$$
v_{k}=\frac{\partial n_{k}}{\partial n}, \quad \sigma_{k}=\frac{\partial s_{k}}{\partial s}, \quad \tau_{k}=\frac{\partial t_{k}}{\partial t} .
$$

Here $\partial / \partial n$ denotes the directional derivative operator along $n^{i}, \partial / \partial s$ denotes the directional operator along $s^{j}$ and $\partial / \partial t$ denotes the directional derivative operator along $t^{j}$.

Following Coburn [1] we shall express the basic relation (3.8) in terms of the characteristic variables. In (3.8) we substitute for $u_{*}^{j}$ from (3.11) and decompose $g^{j k}$ in the form

and get

$$
g^{j k}=t^{j} t^{k}+n^{j} n^{k}+s^{j} s^{k},
$$

$$
c_{*} b_{*}\left(\frac{\partial c_{*}}{\partial t}+\frac{\partial b_{*}}{\partial n}\right)+\left(u_{*}^{2}-2 c_{*}^{2}\right) \frac{\partial b_{*}}{\partial t}+c_{*}^{2} b_{*} t_{k}\left(\nu^{k}+\sigma^{k}\right)-c_{*}^{3} \bar{M}=0 .
$$

Here $\bar{M}$ is the mean curvature of the characteristic manifolds given by

$$
\bar{M}=g^{i j} \Omega_{i j},
$$

$\Omega_{i j}$ being the second fundamental tensor of the characteristic manifolds. The relation (4.2) is the desired intrinsic form of the basic relation (3.8).

To express intrinsic conditions for rotational (or irrotational) motion in terms of characteristic variables, we shall consider the following Bernoulli equation, which is the consequence of (2.2), (2.3) and (2.4)

$$
u^{2}+2 \frac{g(p)}{S(\eta)}=a^{2},
$$

where $a$ is the ultimate velocity magnitude and 


$$
g(p)=\int_{0}^{p} \frac{d x}{P(x)}=\frac{\gamma}{\gamma-1} p^{(\gamma-1) / \gamma} .
$$

The equation (4.4) by use of (3.1) becomes

$$
u_{*}^{2}+2 \frac{g(p)}{S\left(\eta_{*}\right)}=a_{*}^{2}
$$

where $a_{*}$ is the ultimate velocity magnitude in the reduced system.

If $e^{i j k}$ denotes the permutation tensor, then the vorticity vector $w^{i}$ is defined by

$$
w^{i}=e^{i j k} \partial_{j} u_{k} .
$$

Substitution for $u_{k}$ in the above equation from the first relation of (3.1), (4.7) yields

$$
w^{i}=\alpha w_{*}^{i}+e^{i j k}\left(\partial_{j} \alpha\right) u_{* k},
$$

where $w_{*}^{i}$ is the vorticity vector of the flow $F_{*}$. The Lamb vector

$$
\lambda^{i}=e^{i j k} u_{j} w_{k}
$$

transforms to

$$
\lambda_{i}=\lambda_{* i}+u^{2} \partial_{i}(\ln \alpha),
$$

where $\lambda_{*}^{i}$ is the Lamb vector in the $F_{*}$-system. For a complex-screw motion, that is, if the flow has $u_{*}^{i}$ parallel to $w_{*}^{i}$, then $\lambda_{*}^{i}$ is zero and the above relation becomes

$$
\lambda_{i}=u^{2} \partial_{i}(\ln \alpha)
$$

In terms of the Lamb vector, the equations of motion (2.2), by use of (3.1) and (3.2) can be written in the form

$$
2 \lambda_{* i}+\partial_{i} u_{*}^{2}+\left(a_{*}^{2}-u_{*}^{2}\right) \frac{g^{\prime}}{g} \partial_{i} p=0 .
$$

For a complex-screw motion admitting a constant Bernoulli function $a_{*}$, the equation (4.12) implies

$$
a_{*}^{2}-u_{*}^{2}=D g
$$

where $D$ is an arbitrary constant. Substituting this result in (4.6) we find that the motion is isentropic in the $F_{*}$-system. In the $F$-system (4.13) implies

$$
u^{2}=\alpha^{2}\left(a_{*}^{2}-A p^{(\gamma-1) / \gamma}\right)
$$


where $A$ and $a_{*}$ are constants. Since $\lambda_{*}^{i}$ and $u_{*}^{i}$ are orthogonal, multiplying (4.12) by the right hand side of (3.15), we obtain

$$
\left(c_{*} \frac{\partial}{\partial n}+b_{*} \frac{\partial}{\partial t}\right) u_{*}^{2}+\left(a_{*}^{2}-u_{*}^{2}\right) \frac{g^{\prime}}{g}\left(c_{*} \frac{\partial}{\partial n}+b_{*} \frac{\partial}{\partial t}\right) p=0 .
$$

To express (4.12) in the intrinsic form, we take the scalar product of this relation with $t^{i}, n^{i}$ and $s^{i}$ and follow a method very similar to that of Coburn [1] to get

$$
\begin{aligned}
\frac{\partial u_{*}^{2}}{\partial t}+\left(a_{*}^{2}-u_{*}^{2}\right) \frac{g^{\prime}}{g} \frac{\partial p}{\partial t}=2 c_{*}\left(\frac{\partial c_{*}}{\partial t}-\frac{\partial b_{*}}{\partial n}-b_{*} \Omega_{j k} t^{j} t^{k}-c_{*} t^{k} v_{k}\right) \\
\frac{\partial u_{*}^{2}}{\partial n}+\left(a_{*}^{2}-u_{*}^{2}\right) \frac{g^{\prime}}{g} \frac{\partial p}{\partial n}=-2 b_{*}\left(\frac{\partial c_{*}}{\partial t}-\frac{\partial b_{*}}{\partial n}-b_{*} \Omega_{j k} t^{i} t^{k}-c_{*} t^{k} v_{k}\right) \\
\frac{\partial u_{*}^{2}}{\partial s}+\left(a_{*}^{2}-u_{*}^{2}\right) \frac{g^{\prime}}{g} \frac{\partial p}{\partial s}=2 u_{*} \frac{\partial u_{*}}{\partial s}-2\left(b_{*}^{2} \tau_{k}+c_{*}^{2} \nu_{k}\right) s^{k} \\
-2 c_{*} b_{*} n^{j} s^{k}\left(\partial_{j} t_{k}-\partial_{k} t_{j}\right) .
\end{aligned}
$$

Evidently (4.17) is a consequence of (4.15) and (4.16). The equations (4.15), (4.16), (4.18) and (4.2) are the desired intrinsic form of the generalized characteristic relations. If $\alpha \equiv 1$, the equations $(4.2)$ and (4.15) reduce immediately to two of the characteristic relations derived by Coburn $[1]$. The relations $(4.16)-(4.18)$ can be expressed in terms of entropy and enthalpy in the case when $\alpha \equiv 1$, and therefore these relations also reduce to the characteristic relations derived by Coburn [1].

\section{A class of space flows}

Here we shall consider the case that the normals to the characteristic curves $n^{i}$ are along a family of circular helices, and the bicharacteristic curves are along the binormal direction of the $n^{j}$ congruence and $s^{j}$ along the principal normal direction of the $n^{j}$ congruence.

We introduce the cylindrical coordinates, $r, \theta, z$ and write

$$
\begin{aligned}
& n_{i}=\theta_{i} \sin \beta+z_{i} \cos \beta \\
& s_{i}=-r_{i} \\
& t_{i}=-\theta_{i} \cos \beta+z_{i} \sin \beta
\end{aligned}
$$

where $\theta_{i}$ and $z_{i}$ are unit vectors along the increasing $\theta$ and $z$ direction respectively, and $r_{i}$ is the unit vector along the radius of the cylinder; $\beta$ is the angle of the helices and is in general a function of $r$.

Since helices form a congruence of parallel curves on the cylinder, $\bar{M}=\partial_{i} n^{i}=0,[11]$. We are assuming that there exist $\infty^{1}$ family of surfaces 
orthogonal to the congruence $n_{i}$. This condition implies that

$$
e^{i j k} n_{i} \partial_{j} n_{k}=0,
$$

which can be written in the form

$$
t^{k} \frac{\partial n_{k}}{\partial s}-s^{k} \frac{\partial n_{k}}{\partial t}=\mathbf{0}
$$

Substituting for $t^{k}, n^{k}$ and $s^{k}$ from (5.1) into the above equation we find that

$$
\frac{d \beta}{d r}+\frac{\sin \beta \cos \beta}{r}=0
$$

or, on integration,

$$
\cot \beta=r / \xi
$$

where $\xi$ is a constant. We also find that

$$
\begin{aligned}
& v_{k}=-\frac{\sin ^{2} \beta}{r} r_{k}, \quad \sigma_{k}=0, \quad \tau_{k}=-\frac{\cos ^{2} \beta}{r} r_{k}, \\
& \Omega_{j k} t^{j} t^{k}=\partial_{j} n_{k} t^{j} t^{k}=0, \quad \bar{M}=0,
\end{aligned}
$$$$
n^{j} s^{k}\left(\partial_{j} t_{k}-\partial_{k} t_{j}\right)=\beta^{\prime}-\frac{\sin \beta \cos \beta}{r}=-\frac{2 \xi}{\xi^{2}+r^{2}},
$$

$$
\frac{\partial}{\partial n}=\frac{\sin \beta}{r} \frac{\partial}{\partial \theta}+\cos \beta \frac{\partial}{\partial z},
$$

$$
\frac{\partial}{\partial s}=-\frac{\partial}{\partial r}
$$

$$
\frac{\partial}{\partial t}=-\frac{\cos \beta}{r} \frac{\partial}{\partial \theta}+\sin \beta \frac{\partial}{\partial z} .
$$

When these are substituted into (4.2), (4.15), (4.16) and (4.18), the latter become

$$
\begin{gathered}
c_{*} b_{*}\left(\frac{\partial c_{*}}{\partial t}+\frac{\partial b_{*}}{\partial n}\right)+\left(u_{*}^{2}-2 c_{*}^{2}\right) \frac{\partial b_{*}}{\partial t}=0 \\
\left(c_{*} \frac{\partial}{\partial n}+b_{*} \frac{\partial}{\partial t}\right) u_{*}^{2}+\left(a_{*}^{2}-u_{*}^{2}\right) \frac{g^{\prime}}{g}\left(c_{*} \frac{\partial}{\partial n}+b_{*} \frac{\partial}{\partial t}\right) p=0 \\
\frac{\partial u_{*}^{2}}{\partial t}+\left(a_{*}^{2}-u_{*}^{2}\right) \frac{g^{\prime}}{g} \frac{\partial p}{\partial t}=2 c_{*}\left(\frac{\partial c_{*}}{\partial t}-\frac{\partial b_{*}}{\partial n}\right), \\
\frac{\partial u_{*}^{2}}{\partial r}+\left(a_{*}^{2}-u_{*}^{2}\right) \frac{g^{\prime}}{g} \frac{\partial p}{\partial r}=2 u_{*} \frac{\partial u_{*}}{\partial r}-\frac{2}{r}\left(b_{*}^{2} \cos ^{2} \beta+c_{*}^{2} \sin ^{2} \beta\right) \\
+4 c_{*} b_{*} \frac{\xi}{\xi^{2}+r^{2}},
\end{gathered}
$$


where $\partial / \partial n$ and $\partial / \partial t$ are given by (5.4) and (5.6). From (3.11) and (5.1) the stream lines are given by the equations

$$
\frac{d r}{0}=\frac{r d \theta}{c_{*} \sin \beta+b_{*} \cos \beta}=\frac{d z}{-c_{*} \cos \beta+b_{*} \sin \beta} .
$$

Therefore the stream lines lie on the cylinders. $\sin \beta$ and $\cos \beta$ in the equations (5.7) $-(5.10)$ are given by (5.2). As $r$ changes from zero to infinity, $\beta$ changes from $\pi / 2$ to zero.

In particular, if the flow is complex - screw and if $u_{*}$ is a function of $r$ only, then the equations $(5.7)-(5.9)$ are identically satisfied and (5.10) reduces to

$$
u_{*} \frac{\partial u_{*}}{\partial r}-\frac{1}{r}\left(b_{*}^{2} \cos ^{2} \beta+c_{*}^{2} \sin ^{2} \beta\right)+2 b_{*} c_{*}\left(\frac{\xi}{\xi^{2}+r^{2}}\right)=0,
$$

which from (5.2) becomes

$$
u_{*} \frac{d u_{*}}{d r}=\frac{1}{r\left(\xi^{2}+r^{2}\right)}\left[r b_{*}-\xi c_{*}\right]^{2}
$$

and the pressure is given by (4.14). A number of flows can be found to satisfy the above equation. The velocity vector, from (3.11) and (5.1) can be written

$$
u_{*}^{i}=\left(x_{*} \sin \beta+b_{*} \cos \beta\right) \theta^{i}+\left(b_{*} \sin \beta-c_{*} \cos \beta\right) z^{i}
$$

Since

$$
w_{*}^{i}=e^{i j k} \partial_{j} u_{* k}
$$

the vorticity vector $w_{*}^{i}$ by use of (3.11) and (5.1) becomes

$$
\begin{aligned}
w_{*}^{i}= & {\left[\left(\frac{c_{*} \sin ^{2} \beta}{r}-\frac{d c_{*}}{d r}\right) \cos \beta-\left(\frac{b_{*} \cos ^{2} \beta}{r}-\frac{d b_{*}}{d r}\right) \sin \beta\right] \theta^{i} } \\
& -\left[\left(\frac{c_{*} \sin ^{2} \beta}{r} \frac{d c_{*}}{d r}\right) \sin \beta+\left(\frac{d b_{*}}{d r}+b_{*} \frac{\cos ^{2} \beta}{r}\right) \cos \beta\right] z^{i}
\end{aligned}
$$

Let us now transform $u_{*}^{i}, c_{*}, w_{*}^{i}$ to the $F$-system. Since $\alpha$ is constant along the stream line, it satisfies

$$
\left[c_{*}\left(\frac{\sin \beta}{r} \frac{\partial}{\partial \theta}+\cos \beta \frac{\partial}{\partial z}\right)+b_{*}\left(-\frac{\cos \beta}{r} \frac{\partial}{\partial \theta}+\sin \beta \frac{\partial}{\partial z}\right)\right] \alpha=0
$$

The velocity components of the flow $F$ are

$$
u^{i}=\alpha u_{*}^{i}
$$

where $\alpha$ is given by (5.14) and $u_{*}^{i}$ are given by (5.12). The vorticity vector 
$w^{i}$ is given by $(4.8),(5.13)$ and $(5.14)$. The class of flows are rotational.

In particular if $b_{*}=c_{*}$ and $a^{*}$ is a constant, we find from (5.11) and (5.12) that

$$
u_{*}^{2}=2 A r \exp \left[-\frac{2}{\xi} \tan ^{-1}(r / \xi)\right]=2 c_{*}^{2}
$$

and

$$
u_{*}^{i}=\frac{c_{*}}{\sqrt{\xi^{2}+r^{2}}}\left[(\xi-r) \theta^{i}+(\xi+r) z^{i}\right]
$$

where $A$ is a constant. The pressure is given by (4.14). From (5.14) $\alpha$ now satisfies the equation

$$
\left(\frac{1}{r} \frac{\partial}{\partial \theta}+\frac{\partial}{\partial z}\right) \alpha=0
$$

the solution of which is

$$
\alpha=f(z-\theta r) .
$$

In the $F$-system the components of velocity are given by

$$
u^{i}=\frac{\alpha c_{*}}{\sqrt{\xi^{2}+r^{2}}}\left[(\xi-r) \theta^{i}+(\xi+r) z^{i}\right]
$$

where $c_{*}$ is given by (5.15) and $\alpha$ by (5.16). From (4.14) and (5.15) the pressure is given by

$$
p^{(\gamma-1) / \gamma}=u_{*}^{2}-a_{*}^{2} .
$$

The entropy in the $F$-system now becomes

$$
S(\eta)=\frac{1}{\alpha^{2}} S_{*},
$$

where $S_{*}$ is a constant. In (5.17) and (5.18) $u_{*}$ and $\alpha$ are given by (5.15) and (5.16) respectively. For a polytropic gas, the equations of motion and continuity assume the form

$$
\begin{aligned}
S p^{1 / \gamma} u^{j} \partial_{j} u_{i}+\partial_{i} p & =0, \\
\partial_{j}\left(p^{1 / \gamma} u_{j}\right) & =0 .
\end{aligned}
$$

Transforming the above equations into polar coordinates, we find that the above components of velocity and the above pressure and entropy satisfy the transformed equations. From (5.13) the vorticity vector can be obtained.

Other classes of flows can be obtained by assigning appropriate functions to either $b_{*}$ and $c_{*}$ in the equation (5.11).

Now let us consider the case when $t^{j}$, the bicharacteristic direction, 
is along $r^{j}$ the radius of the cylinder, so that $n^{j}, t^{j}$ and $s^{j}$ form a right-handed triple at each point. That is, let

$$
\begin{aligned}
n_{i} & =\theta_{i} \sin \beta+z_{i} \cos \beta \\
t_{i} & =-r_{i} \\
s_{i} & =-\theta_{i} \cos \beta+z_{i} \sin \beta .
\end{aligned}
$$

We find the following relations hold:

$$
v_{k}=-\frac{\sin ^{2} \beta}{r} r_{k}, \quad \tau_{k}=0, \quad \sigma_{k}=-\frac{\cos ^{2} \beta}{r} r_{k},
$$

and

$$
\begin{aligned}
& \Omega_{j k} t^{j} t^{k}=\partial_{j} n_{k} t^{j} t^{k}=0, \\
& n^{j} s^{k}\left(\partial_{j} t_{k}-\partial_{k} t_{j}\right)=0,
\end{aligned}
$$

By virtue of the above relations, (4.2), (4.15), (4.16), and (4.18) now reduce respectively to

$$
\begin{aligned}
& c_{*} b_{*}\left(-\frac{\partial c_{*}}{\partial r}+\frac{\partial b_{*}}{\partial n}\right)-\left(u_{*}^{2}-2 c_{*}^{2}\right) \frac{\partial b_{*}}{\partial r}+c_{*}^{2} b_{*}=0 \\
& \left(c_{*} \frac{\partial}{\partial n}-b_{*} \frac{\partial}{\partial r}\right) u_{*}^{2}+\left(a^{2}-u_{*}^{2}\right) \frac{g^{\prime}}{g}\left(c_{*} \frac{\partial}{\partial n}-b_{*} \frac{\partial}{\partial r}\right) p=0 \\
& -\frac{\partial u_{*}^{2}}{\partial r}-\left(a_{*}^{2}-u_{*}^{2}\right) \frac{g^{\prime}}{g} \frac{\partial p}{\partial r}=2 c_{*}\left(-\frac{\partial c_{*}}{\partial r}-\frac{\partial b_{*}}{\partial n}-c_{*} \frac{\sin ^{2} \beta}{r}\right)
\end{aligned}
$$

$$
\left(a_{*}^{2}-u_{*}^{2}\right) \frac{g^{\prime}}{g} \frac{\partial p}{\partial s}=0
$$

where $\partial / \partial n$ and $\partial / \partial s$ are given by the relations (5.20) and (5.21). The last result shows that the pressure does not vary along the $s^{j}$ direction. From (3.11) and (5.18) the differential equations of the streamlines are

$$
-\frac{d r}{b_{*}}=\frac{r d \theta}{c_{*} \sin \beta}=\frac{d z}{c_{*} \cos \beta} \text {. }
$$

In this case also $\sin \beta$ and $\cos \beta$ are given by (5.2). As $r$ changes from zero to infinity $\beta$ changes from $\pi / 2$ to zero. 
In particular, if the flow is complex-screw and if $u_{*}$ is a function of $r$ only, then the equations (5.23) and (5.25) are identically satisfied, and (5.22) and (5.24) become

$$
\begin{aligned}
& c_{*} b_{*} \frac{\partial c_{*}}{\partial r}-\left(u_{*}^{2}-2 c_{*}^{2}\right) \frac{d b_{*}}{d r}+\frac{1}{r} c_{*}^{2} b_{*}=0, \\
& \frac{1}{c_{*}} \frac{\partial c_{*}}{\partial r}=-\frac{\sin ^{2} \beta}{r} .
\end{aligned}
$$

Substituting for $\sin \beta$ from (5.2) into the above equation, we find that

$$
c_{*}^{2}=B^{2} \frac{\xi^{2}+r^{2}}{r^{2}}
$$

$B$ being an arbitrary constant. Eliminating $\left(d c_{*} / d r\right)$ from (5.27) and (5.28) we get

$$
\left(\frac{b_{*}^{2}}{c_{*}^{2}}-1\right) \frac{1}{b_{*}} \frac{d b_{*}}{d r}=\frac{1}{r} \cos ^{2} \beta
$$

Substituting for $\cos \beta$ from (5.2) and for $c_{*}$ from (5.29) we find the above result becomes

$$
\left[B^{2} \frac{\xi^{2}+r^{2}}{r^{2}}-b_{*}^{2}\right] \frac{1}{b_{*}} \frac{d b_{*}}{d r}=-\frac{B^{2}}{r}
$$

the solution of which is

$$
b_{*}=B \frac{\xi}{r} .
$$

From (3.11), (3.12), (5.18), and (5.28) the velocity and its magnitude are given by

$$
u_{*}^{i}=\frac{B}{r}\left[-\xi r^{i}+\xi \theta^{i}+r z^{i}\right]
$$

and

$$
u_{*}^{2}=\frac{B^{2}}{r^{2}}\left(2 \xi^{2}+r^{2}\right)
$$

From (5.2), (5.26), (5.29) and (5.30) the differential equations of the stream lines are

$$
\frac{d r}{\xi}=\frac{-r d \theta}{\sqrt{\xi^{2}+r^{2}}}=\frac{-d z}{\sqrt{\xi^{2}+r^{2}}} .
$$

These equations can be integrated. The stream lines lie on the spiral surface $z=r \theta+$ constant. 
The Mach number, $M$, of the flow is given by

$$
M^{2}=\frac{u_{*}^{2}}{c_{*}^{2}}=\frac{2 \xi^{2}+r^{2}}{\xi^{2}+r^{2}} .
$$

Thus $M$ decreases as $r$ increases and it approaches $I$ as $r$ approaches infinity. The vorticity vector $w_{*}^{i}$ reduces to zero in this case and therefore the motion is irrotational.

In the $F$-system the components of velocity are given by

$$
u^{i}=\alpha u_{*}^{i}
$$

where, $\alpha$ now satisfies the equation

$$
\left(-\frac{\partial}{\partial r}+\frac{\partial}{\partial \theta}+\frac{r}{\xi} \cdot \frac{\partial}{\partial z}\right) \alpha=0 .
$$

The solution of the above equation is

$$
\alpha=\psi\left(r+\theta, \xi z+\frac{1}{2} r^{2}\right),
$$

$\psi$ is an arbitrary function. The vorticity vector in the $F$-system is given by (4.8), (5.30) and (5.31). We find that the motion is rotational. The pressure, the components of velocity and entropy in the $F$-system are

$$
\begin{aligned}
p^{(\gamma-1) / \gamma} & =\frac{B^{2}}{r^{2}}\left(2 \xi^{2}+r^{2}\right)+a_{*}^{2}, \\
u^{i} & =\frac{\alpha B}{r}\left[-\xi r^{i}+\xi \theta^{i}+r z^{i}\right], \\
S(\eta) & =\frac{1}{\alpha^{2}} S_{*},
\end{aligned}
$$

where $a_{*}, S_{*}, B, \xi$ are constants and $\alpha$ is given by (5.32). It can be verified that these results satisfy the equations of motion and continuity in polar coordinates identically.

Both classes of flows are non-isentropic in the $F$-system.

\section{Acknowledgment}

This work was done while the author held a visiting research position at the University of Queensland. He takes this opportunity to thank the University for support and Professor A. F. Pillow for providing a stimulating research environment. 


\section{References}

[1] N. Coburn, 'Intrinsic form of the characteristic, relations in the steady supersonic flow of a compressible fluid', Quarterly Journal of Applied Mathematics 15 (1957), 237-248.

[2] C. Truesdell, and R. Toupin, The Classical Field Theories (Handbuch der Physik, Band III, Springer Verlag (1960), 416).

[3] R. C. Prim, 'Steady rotational flow of ideal gases', J. Rat. Mech. Anal. 1 (1952), 425-497.

[4] P. Neményi and R. Prim, 'Some geometrical properties of plane gas flows', J. Maths. Physics 27 (1948), 130-135.

[5] A. G. Hansen and M. H. Martin, 'Some geometrical properties of plane flows,' Proc. Camb. Phil. Soc. 47 (1951), 763-776.

[6] P. Smith, 'Some intrinsic properties of spatial gas flows', J. Maths. Mech. 12 (1963), 27-32.

[7] P. Smith, 'The steady magnetohydrodynamic flow of perfectly conducting fluids', J. Math. Mech. 12 (1963), 505-520.

[8] C. E. Weatherburn, Differential Geometry, I. (Cambridge University Press (1955), 146).

[9] N. Coburn, 'Intrinsic relations satisfied by the velocity and vorticity vectors', Michigan Math. J. 1 (1952), 113-130.

[10] N. Coburn, 'Discontjnuities in compressible fluid flows', Maths. Mag. 27 (1954), 245-264.

[11] C. E. Weatherburn, Differential Geometry, I. (Cambridge University Press (1955), 258).

University of Queensland, Australia

University of Rhode Island, U.S.A. 